\title{
A ENFERMAGEM NA PERDA DA ACUIDAdE VISUAL CAUSADA POR TRIQUÍASE EM PACIENTES DE HANSENIASE
}

\author{
Selma Regina Axcar Salotti * \\ Hannelore Vieth ** \\ Sérgio Passeroti ***
}

\begin{abstract}
RESUMO - O reconhecimento do grande número de pacientes com triquíase e a inexistência de publicações nesse ramo motivou os autores a elaborar o presente trabalho. Os autores apresentam as causas, sinais, sintomas e consequências da triquíase em relação à córnea e acuidade visual, como também o tratamento com técnicas simples. Pelos resultados obtidos durante os oito anos de experiência, verificou-se a grande valia do tratamento na prevenção da perda da acuidade visual provocada pela triquiase.

ABSTRACT - The knowledge of a great number of patients with trichiasis and the lack of existence of publication about this subject, has motivate the authors to elaborate this article. The authors intenal to show the causes the signs, symptoms and consequences of trichiasis in relation to the cornea and visual acuity, as well as the treatment using simple thecniques. By the results obtained during the 8 years of experience, it has been noticed the great value of the treatment on prevention of loss of visual acuity caused by trichiasis.
\end{abstract}

\section{INTRODUÇĀO}

\subsection{Histórico}

Desde 1982 o Hospital "Lauro de Souza Lima" dispõe de uma Unidade de Prevenção Oftalmológica. Todos os pacientes internados, e grande parte dos pacientes ambulatoriais passam, de rotina, pela avaliação específica, para detectar as alterações oculares o mais precoce possível.

Atualmente trabalham nessa Unidade, duas enfermeiras em tempo integral e um médico oftalmologista.

Durante o funcionamento da Unidade, chamou a atenção o grande número de pacientes que apresentavam triquíase, muitas vezes, sem queixas especificas, mas produzindo graves lesões oculares.

Aplicando uma técnica simples de remoção manual dos cílios causadores da triquíase, verificou-se uma melhora imediata da sintomatologia, e no decorrer do tempo uma diminuição das infecções secundárias e melhora da acuidade visual.
Os resultados obtidos levaram a equipe a elaborar este trabalho. Pretende-se com o memo, despertar o interesse dos profissionais da área de enfermagem para o problema, sabendo que tal comportamento não ocorre somente na Hanseníase.

\subsection{Objetivos do Trabalho}

1.2.1 Mostrar as principais causas e consequências da triquíase na córnea em pacientes com Hanseníase.

1.2.2 Apresentar técnicas simples para a detecção e a remoção dos cllios.

1.2.3 Mostrar a recuperação do olho (da córnea), após tratamento.

1.2.4 Despertar interesse dos profissionais da área de enfermagem para esse problema.

* Enfermeira da Unidade de Oftalmologia do Instituto "Lauro de Souza Lima" - SP.

* Enfermeira-Professora de Enfermagem da Universidade Livre de Berlim a Serviço da DAHW (Germam Leprosy Relief Association).

*** Médico-Chefe do Departamento de Oftalmologia do Instituto "Lniro de Souza Lima" - SP. 


\subsection{Resumo sobre Hanseníase}

A Haseníase é uma moléstia infecciosa produzida por um bacilo álcool-ácido resistente, o Mycobacterium leprae. A identificação deste bacilo foi feita por Hansen em 1784, e em sua homenagem, leva-lhe o nome. É um bacilo não cultivável até o momento. A reprodução por inoculação em animal, só teve êxito a partir de 1970, quando se passou a usar a tatu dasypus novencinctus como modelo animal. Este animal quando inoculado desenvolve comprometimentos muito semelhantes aos do homem, sobrepujando-os em alguns aspectos.

A Hansenfase é uma doença própria do ser humaro, sendo este o reservatório e a fonte de infecção. Pacientes portadores das formas contagiantes, eliminam bacilos em grande quantidade pelas secreções nasais, orofaríngeas e ulceraçổes da pele. Os bacilos eliminados podem atingir o individuo sadio e penetrar no organismo pelas mucosas e especialmente a nasal ou através da pele lesada. Uma vez penetrada no organismo pode produzir a doença nos individuos susceptiveis.

Depois de um perfodo de incubação que varia de 2 a 5 anos, as primeiras manifestações iniciais são o aparecimento de manchas hipocrômicas, anestésicas e anidróticas em qualquer parte do organismo. Essa fase perdura em média de 3 a 5 anos, é chamada hanseníase indeterminada (I). Dependendo da resistência imunológica do organismo, a infecção pelo bacilo de Hansen, a forma (I) pode ter regressão espontânea com cura completa, ou evoluir para as formas polares Tuberculóides $(\mathrm{T})$ ou Virchovianas (V) ou mesmo para a forma Bordeline (B).

No decorrer da evolução, a hanseníase pode comprometer diversos segmentos do corpo. As partes mais comprometidas são a pele, mucosas e os nervos periféricos. Entretanto, outros órgãos são frequentemente atingidos quer por ação direta do bacilo ou indiretamente nos processos reacionais. $O$ aparelho visual pode ser atingido em ambos os processos.

Os comprometimentos neurológicos são mais frequentes nos membros superiores e membros inferiores, levando a perdas severas da sensibilidade e deficiências motoras. Desta forma, o paciente é privado de um dos mais importantes mecanismos de defesa do organismo que $e$ a capacidade de sentir dor. Á ausência de sensibilidade nas mãos e nos pés exige do paciente um maior uso da visão para proteger-se.

Os comprometimentos oculares conduzem muito frenqüentemente à diminuição da acuidade visual ou mesmo à cegueira. Isso, associado à insensibilidade de mãos e pés expōe o indiví- duo à graves riscos tornando-o vulnerável a toda ordem de traumas, ferimentos e mutilaçōes.

A observação tem demonstrado que os comprometimentos oculares são muito mais freqüentes do que aparentam. Também observa-se que nos programas de controle de tratamento da hanseníase tem-se dado muito pouca atenção a esse aspecto.

\section{MATERIAL E MÉTODOS}

Para o presente trabalho foram examinados, de fevereiro a novembro de 1990, 600 pacientes, sendo 300 de forma Virchoviana, 150 da forma Bordeline e 150 Tuberculóides.

Foram avaliados os sintomas subjetivos (queixas do paciente) e sinais objetivos apresentados pelos mesmos com o auxnlio da lâmpada de fenda e o teste de Fluoresceína.

Todos os pacientes que apresentaram comprometimento, foram submetidos ao tratamento, reavaliados e acompanhados periodicamente.

\section{TRIQUÍASE NA HANSENIASE}

\subsection{Conceito}

O quadro de cnlios invertidos (pelo menos um), roçando a córnea e conjuntiva é chamado triquíase. Estes cilios em geral, muitos finos, sedosos, quase sem cor, são difíceis de serem vistos sem instrumental e técnica apropriada.

\subsection{Causas}

A avaliação de mais de 2.500 pacientes, permitiu observar que a triquíase está associada a:

3.2.1 Entrópio: - É a inversão do bordo palpebral superior ou inferior por cicatrizes (reaçōes hansênicas, nódulos, ulcerações), edema e infiltração ou em consequência da blefarocalase (pregueamento excessivo da pele da pálpebra superior).

3.2.2 Madarose: - É a queda dos pelos da região superciliar e ciliar. As causas são em geral infiltrações hansênicas. Pode ser parcial, difusa ou total. $O$ recrescimento do pelos na região ciliar podem causar grandes problemas, (já que crescem em direção anormal). São muito finos, sedosos, atróficos, quase sem cor, perdendo sua função protetora a muitas vezes ferindo a córnea e conjuntiva.

3.2.3 Nódulos, Infiltrações e Placas: - Durante a fase aguda da Hanseníase ou em surtos reacionais aparecem nódulos, infiltraçōes e placas. Quando surgem na região ciliar, provocam alte- 
ração no posicionamento do bordo palpebral, podendo causar inversão da mesma, triaurase.

\subsection{Sinais e Sintomas da Triquíase}

Os sinais da triquíase a olho desarmado são: hiperemia conjuntival, lacrimejamento, crlios invertidos roçando a córnea.

Pacientes com triquíase costumam queixarse de: lacrimejamento, prurido, sensação de corpo estranho, ardor, às vezes dor e visão embaçada.

Observaçāo: - Estas queixas estão diminuídas ou ausentes em pacientes com hipoestesia e anestesia de córnea (mais ou menos $50 \%$ dos pacientes de Hanseníase).

\subsection{Exame para Deteç̧āo da Triquíase}

3.4.1 Material: - Para facilitar a detecção da triquíase, recomenda-se o uso do seguinte material:

- foco luminoso

- lente de aumento.

3.4.2 Método: - Examinador em pé, atrás do paciente. Paciente sentado, apoia a cabeça no examinador. Esta posição oferece maior apoio e segurança. Com os dedos o examinador afasta delicadamente a pálpebra superior. Com auxnio do foco luminoso e da lente de aumento, examina o bordo palpebral superior e inferior, observando a posição dos ćllios. Se estiverem invertidos é possf́vel visualizar com esse simples exame.

\subsection{Tratamento da Triquiase}

3.5.1 Material: - O material necessário para a remoção dos cílios invertidos é:

- foco luminoso

- lente de aumento - fixado no foco ou lupa binocular

- pinça (tipo sobrancelha ou pinça de íris).

3.5.2 Método: - A posição do paciente e do enfermeiro é a mesma usada para a detecção da triquíase. Pede-se ao paciente que olhe para baixo em caso de triquíase de pálpebra superior e para cima em caso de triquíase da pálpebra inferior.

Com a mão esquerda o enfermeiro segura o foco luminoso com a lente de aumento fixada na mesma, ou lupa binocular e foco. Com o quarto e quinto dedos da mesma mão afasta delicadamente o bordo palpebral. Com a pinça na mão direita arranca um por um os cllios invertidos. Ao tentar remover os mesmos, estes podem romper-se, ficando difícil a remoção. Para não quebrar, é necessário prendê-los com a pinça o mais próximo possível do bordo, com cuidado para não causar traumatismos.

Após cada seção de retirada de cnlios, é indispensável o uso de um lubrificante artificial da córnea. Recomenda-se o uso do mesmo como proteção da córnea em média três vezes por dia. Em casos onde já houve lesões maiores na córnea, há necessidade do uso de pomada, auxiliando na recuperação do epitélio coreano.

3.5.3 Tratamento definitivo: - Existe o tratamento definitivo para a triquíase em forma de epilação e/ou cirurgia plástica. Como a maioria dos pacientes não tem acesso imediato ou tandio a estas técnicas, por motivos diversos, a retirada manual dos cnlios torna-se o único meio acessível para a prevenção da perda da acuidade visual por triquíase.

3.5.4 Orientação ao paciente: - É ponto fundamental na prevenção das lesōes oculares produzidas pela triquíase.

É preciso conseguir que o paciente tome consciência do seu problema e dos riscos que corre. O reconhecimento da sintomatologia é o ponto de partida para um programa de prevenção. Deve-se lembrar que há pacientes que conservam a sensibilidade da córnea o que permite a percepção precoce da presença de cnlios roçando na córnea.

Nesses casos, recomenda-se ao paciente que ao perceber o problema procure o serviço para a remoção dos cllios anômalos.

Os pacientes com hipoestesia ou anestesia corneana, carecem daquele sistema de alarme. Estes, necessitam ser orientados quanto aos sintomas: lacrimejamento, hiperemia, visão embaçada e a necessidade de procurar imediatamente o serviço especializado para avaliaçāo e tratamento, evitando assim as consequências mais graves da triquíase tais como: infecção secundária pela auto contaminação (prurido e lacrimejamento) leucoma (opać́ficais) da córnea, ponoftalmia e cegueira irreversível.

Mesmo que não haja nenhum sintoma objetivo da triquíase, o paciente portador deste problema deve submeter-se a um exame de controle, no mínimo, uma vez por mês.

\subsection{Avaliaçāo da Eficiência do Tratamento}

A diminuição ou desaparecimento dos sinais e sintomas e a melhora da acuidade visual são dados indicativos de acerto da técnica aplicada. A remoção dos cllios tem um efeito imediato sobre os sintomas: desaparece a sensação de corpo estranho, prurido, ardor e dor no olhu. Os sinais na córnea desaparecem normalmente ap6s 24 horas. 


\section{RESULTADOS}

Foram examinados 600 pacientes no total, sendo agrupados pela forma da doença, usando-se a classificação de Madriad: 300 pacientes da forma Virchoviana (50\%); 150 pacientes da forma Tuberculóide (25\%) e 150 pacientes da forma Borderline (25\%).

Dos 300 pacientes examinados da forma Virchoviana, 142 ou seja, $47,3 \%$, dos 150 da forma Tuberculoide (8), ou seja 5,3\%, dos 150 da forma Bordeline (58) ou seja $38,7 \%$, apresentaram triquiase, sendo o número mais significativo na forma Virchoviana 47,3\%, como pode ser visto na Tabela 01.

A Tabela 02 mostra as principais causas da triquíase, separados pela forma clínica da doença. Como pode ser visto nesta, o Entrópio é a

principal causa da triquiase, tanto na forma Virchoviana $(21,7 \%)$ como na forma Borderline $(18,7 \%)$.

Na Tabela 03, vemos o número de pacientes com queixas e sem queixas, separados pela forma clínica da doença, demonstrando que na forma Virchoviana, 65,5\% dos pacientes com triquíase não apresentaram queixas. Esse fato se deve ao grande número de pacientes de Hansenfase que apresentam alterações na sensibilidade da córnea causada pelo comprometimento hansênico do 5o par de nervos craneanos.

Sendo avaliados através do teste de fluoresceĺna e analisando a córnea com auxílio da lâmpada de fenda, foram encontradas micro lesōes epiteliais em todos os pacientes.

Tabela 01 - Número de pacientes com e sem triquíase em relação à forma clínica

\begin{tabular}{|c|c|c|c|c|c|c|c|c|c|}
\hline \multirow[t]{2}{*}{ Forma Clinica } & \multicolumn{3}{|c|}{$\begin{array}{c}\text { № de Pacientes } \\
\text { Examinados }\end{array}$} & \multicolumn{3}{|c|}{$\begin{array}{c}\text { Com } \\
\text { Triquiase }\end{array}$} & \multicolumn{3}{|c|}{$\begin{array}{c}\text { Sem } \\
\text { Triquiase }\end{array}$} \\
\hline & 150 & - & $25 \%$ & 08 & - & $5,3 \%$ & 142 & - & $94,7 \%$ \\
\hline Bordeline & 150 & - & $25 \%$ & 58 & - & 38, & 92 & - & $61,3 \%$ \\
\hline Virchoviana & 300 & - & $50 \%$ & 142 & - & $47,3 \%$ & 158 & - & $52,7 \%$ \\
\hline Total & 600 & - & $100 \%$ & 208 & - & $34,7 \%$ & 392 & - & $65,3 \%$ \\
\hline
\end{tabular}

FONTE: UPO - Instituto "Lauro de Souza Lima"

Bauru - SP - 1990

Tabela 02 - Número de pacientes com triquiase conforme causa e forma clínica

\begin{tabular}{|c|c|c|c|c|c|c|c|c|c|c|c|c|c|}
\hline \multirow[b]{2}{*}{ Tuberculbide } & \multirow{2}{*}{$\begin{array}{c}\begin{array}{c}\text { Pacientes } \\
\text { Examinados }\end{array} \\
150 \\
\end{array}$} & \multicolumn{3}{|c|}{$\begin{array}{l}\text { Pacientes } \\
\text { c/Triquiase } \\
\text { p/Causa de } \\
\text { Entrópio }\end{array}$} & \multicolumn{3}{|c|}{$\begin{array}{l}\text { Pacientes } \\
\text { c/Triquiase } \\
\text { p/Causa de } \\
\text { Madarose }\end{array}$} & \multicolumn{3}{|c|}{$\begin{array}{c}\text { Pacientes } \\
\text { c/Triquiase } \\
\text { p/Causa } \\
\text { Infil. e Nod. }\end{array}$} & \multicolumn{3}{|c|}{$\begin{array}{c}\text { Pacientes } \\
\text { c/Triquilase } \\
\text { p/Outras } \\
\text { Causas }\end{array}$} \\
\hline & & & - & & 02 & - & $1,3 \%$ & 03 & - & $2,0 \%$ & 03 & - & $2,0 \%$ \\
\hline Borderline & 150 & 28 & - & $18,7 \%$ & 12 & - & $8,0 \%$ & 16 & - & $10,7 \%$ & 02 & - & $1,3 \%$ \\
\hline Virchoviana & 300 & 65 & - & $21,7 \%$ & 57 & - & $19,0 \%$ & 19 & - & $6,4 \%$ & 01 & - & $0,3 \%$ \\
\hline Total & 600 & 93 & - & $15,5 \%$ & 71 & - & $11,8 \%$ & 38 & - & $6,3 \%$ & 06 & - & $1,0 \%$ \\
\hline
\end{tabular}

FONTE: UPO - Instituto "Lauro de Souza Lima"

Bauru - SP - 1990

Tabela 03 - Número de pacientes com triquíase em relação as queixas subjetivas ou não e forma clínica

Forma Clinica

\begin{tabular}{|c|c|c|c|c|c|c|c|c|c|}
\hline Forma Clinica & & m & jeixas & Se & $\mathrm{c}$ & eixas & & To & \\
\hline Tuberculóide & 07 & - & $87 \%$ & 01 & - & $12,5 \%$ & 08 & - & $100 \%$ \\
\hline Bordeline & 30 & - & $51,7 \%$ & 28 & - & $48,3 \%$ & 58 & - & $100 \%$ \\
\hline Virchoviana & 49 & - & $34,5 \%$ & 93 & - & $65.5 \%$ & 142 & - & $100 \%$ \\
\hline Total & 86 & - & $41,3 \%$ & 122 & - & $58,7 \%$ & 208 & - & $100 \%$ \\
\hline
\end{tabular}

\section{CONCLUSĀO}

$\mathrm{Na}$ avaliação dos pacientes de Hansenfase deve-se incluir, de rotina, a varificação da existência de triquíase. Sendo a detecção fácil e requerendo apenas poucos recursos materiais e técnicos, todo posto de atendimento de paciente de Hanseníase, deve fazer avaliação ocular pelo menos cada 6 (seis) meses. Uma vez detectado o problema, o paciente deve ser orientado e submetido "à retirada dos cnlios. Agendado para retorno e orientado para procurar auxflio, cada vez que sente a córnea sendo irritada.

Sabendo que mais de $50 \%$ dos pacientes apresentam, além da triquíase, alteração na sen- 
sibilidade da córnea, esta tem que ser avaliada. Caso o paciente apresente uma sensibilidade baixa ou ausente, a retirada de cnlios deve ser rotina uma vez por mês independente de o paciente apresentar sintomas ou não.

O presente trabalho foi de grande valia para conscientização dos pacientes que passaram a procurar sistematicamente o serviço para a remoção dos cllios com a técnica simples aqui apresentada. Notou-se uma diminuição das lesões e infecções do epitélio corneano causadas pela triquíase, e que esta técnica é um dos meios de prevenir as lesões e a destruição do epitélio corneano, a diminuição progressiva da visão e até infecções secundárias, que chegam algumas vezes à cegueira.

\section{RECOME NDAÇÕES}

6.1 Que todo paciente de Hansenfase seja rotineiramente avaliado em busca de sinais de triquíase.

6.2 Que os Centro de Saúde, Hospitais e Postos de atendimento providenciem pessoal treinado e material necessário para o tratamento preventivo do paciente com triqurase.

6.3 Que todos os profissionais procurem atualizar seus conhecimentos na técnica de detecção e tratamento da triquiase, tendo em vista que a mesma não acomete somente pacientes de Hanseníase.

\section{REFERÊNCIAS BIBLIOGRÁFICAS}

1 ALLEN, J.H. et alli. The Pathology of Ocular Leprosy. Archives of Ophthalmology, 64: 216-220, 1960.

2 ALLEN, J.H. The Pathology of Ocular Leprosy. American Joumal of Ophthalmology, 61: 987-992, 1966.

3 BECHELLI, L.M., ROTHBERG,:A. Compêndio de Leprologia. Rio de Janeiro, Ministério da Saúde. 1956, 319, 324.

4 BRAND, M. The Car of the Eyes. The Star, 37: 1, 2, 3, 4 , 1978.

5 BRASIL, Ministério da Saúde. Prevenção e Tratamento das Incapacidades Fisicas Mediante Técnicas Simples. Rio de Janeiro, 1977.

6 - Manual de normas e procedimentos da enfermagem do tipo ambulatorial no Controle da Hansenfase. Brasília, 1978.

7 . Guia de consulta de enfermagem no controle da hansentase. Brasília, 16, 1979.

8 . Guia para o contrôle da hansenfose. Brasília, 2a edição, 1984.

9 CENTRO DE ESTUDOS “DR. REYNALDO QUAGLIATO”. Reabilitação em Hansenfase e Prevenção de Incapacidades. 4 ed. Bauru, Hospital "Lauro de Souza Lima", 1982. p.55-60.

10 CRISTOFOLINI, L. Prevenção de Incapacidade na Hanseníase. In: Revista Brasileira de Enfermagem. 35: 226-237, 1982.

11 . Assistência de Enfermagem na hanseníase. Salusvita, 4 (1): 01-09, Bauru, 1985.
12 CRISTOFOLINI, L. et alli. A enfermagem na prevençāo e tratamento dos comprometimentos oculares na hansenfase. CBEn - São Paulo, 1983 (não publicado).

13 . Açōes da enfermagem nos comprometimentos oculares na hanseníase. In: Saluvira, 5 (1) 37-51, Bauru, 1987.

14 . Rotina de Enfermagem na avaliaçāo dos comprometimentos oculares na Hanseníase. Revista Brasileira de Enfermagem, 39: 86-89, 1986.

15 CHOYCE, D.P. Diagnosis and Management of Ocular Leprosy. Brit.J. Ophthal., 53, 217-223, 1969.

16 GONÇALVES, A. Epidemiologia e controle da hansenfase no Brasil. Brasília, MS, DNDS, 1983.

17 . Aspectos da epidemiologia e controle da harsenfase no Brasil. Brasília, 1984 (não publicado).

18 MALLA, BRAND, ANTEN. Ocular Findings in Leprosy Petients in an Institution in Nepal (Klokana). Brit. J. Ophthal, 65,: 226-230, 1981.

19 MENDONÇA DE BARROS, J. Aspectos Clínicos do comprometimento Oftalmológico. Melhoramentos, São Paulo, 1939.

20 MENDONÇA DE BARROS, J. As complicaçōes oculares na Lepra. Revista Brasileira de Leprologia, 14: 103-134, 1945.

21 VALE, S. Subsidios para o estudo da lepra ocular. Imprensa Nacional, Rio de Janeiro, 1944.

22 VIETH, H. et alli. A valiaçōes e tratamento o olho seco na Hansenfase. Revista Brasileira de Enfermagem, 40: 118-122, 1987. 